\title{
Symptomatic paroxysmal atrial fibrillation in a patient with unilateral pulmonary vein atresia
}

\author{
Sharmila Sehli MD, David M Donaldson MD, FACC
}

\begin{abstract}
A 52-year-old man with symptomatic paroxysmal atrial fibrillation was offered an atrial fibrillation (AF) ablation procedure. His echocardiogram indicated that he had no structural heart disease. A cardiac computed tomographic (CT) scan showed enlargement of the right pulmonary veins, absence of the left pulmonary veins, a prominent left atrial appendage, and a hypoplastic left lung. Cardiac CT with an electroanatomic mapping system confirmed a prominent left atrial appendage and the absence of the left pulmonary veins. Due to the limited number of patients with this condition, information about ablation remains very limited, and his ablation was deferred. Unilateral pulmonary vein atresia is a rare condition in adults which results from failure of incorporation of the common pulmonary vein into the left atrium. This case demonstrates the clinical importance of preprocedural imaging prior to AF ablation.
\end{abstract}

Key words: Atrial fibrillation, pulmonary vein atresia

\section{Case Presenttion}

A 52-year-old man with a history of paroxysmal atrial fibrillation (PAF), atrial flutter, hypertension, and hyperlipidemia was referred for evaluation of symptomatic PAF. He had worsening dyspnea on exertion and palpitations associated with episodes of PAF. He was treated with flecainide and a calcium channel blocker without significant improvement in his symptoms and was offered an atrial fibrillation (AF) ablation. A preprocedural chest X-ray revealed an elevated left hemithorax and reduced left lung volume but no cardiomegaly. Transesophageal echo-

Corresponding author: Sharmila Sehli MD

Contact Information: sharmilasehli@gmail.com

DOI: 10.12746/swrccc2015.0309.121 cardiogram prior to AF ablation revealed no significant structural heart disease with a $4 \mathrm{~cm}$ left atrium, a prominent left atrial appendage with normal velocities, and no thrombus. The estimated systolic pulmonary pressure on Doppler examination was normal. A cardiac computed tomographic (CT) angiogram showed enlargement of the right pulmonary veins, absence of the left pulmonary veins, bronchial artery collateralization to the left lung, a prominent left atrial appendage, a small left pulmonary artery, and a hypoplastic left lung with interstitial thickening. A cardiac CT with an electroanatomic mapping system showed a prominent left atrial appendage and the absence of the left pulmonary veins (Figure1). 

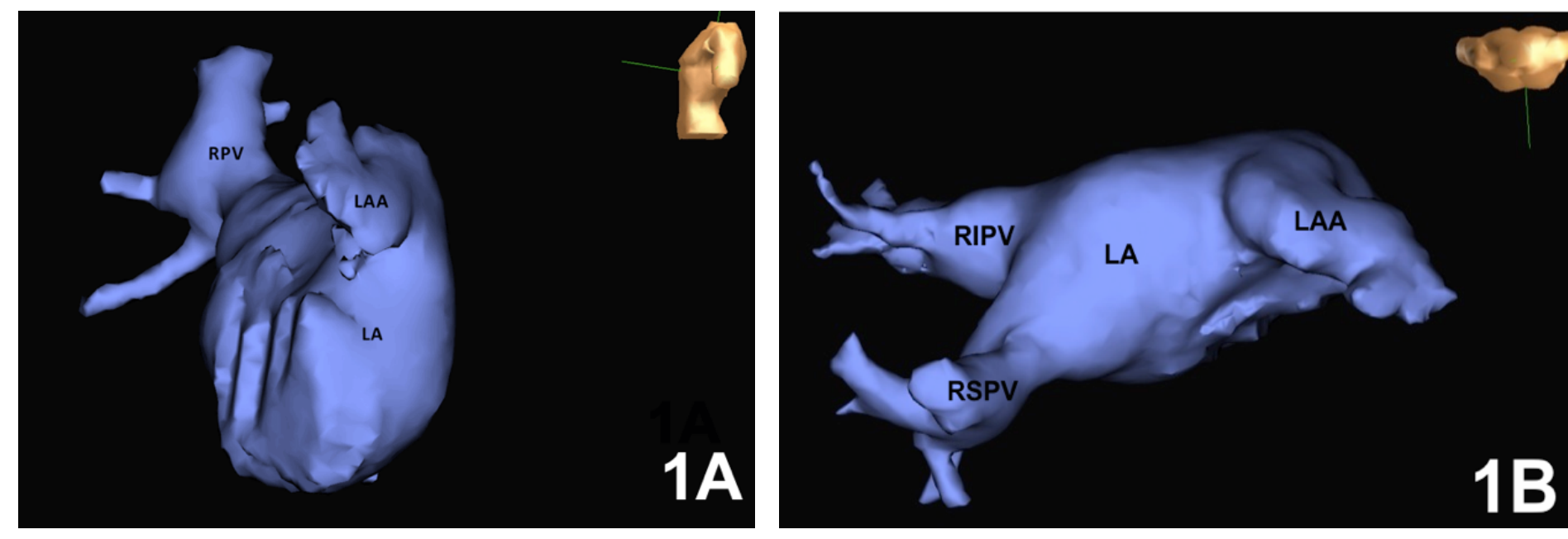

Figure 1. Cardiac CT with an electroanatomic mapping system; 1A- left lateral view; 1B- top down view; LA-left atrium; LAA-left atrial appendage; RPV-right pulmonary vein; RIPV-right inferior pulmonary vein; RSPV-right superior pulmonary vein

\section{Discussion}

Unilateral pulmonary vein atresia is a very rare condition in adults. It likely results from the failure of incorporation of the common pulmonary vein into the left atrium and is associated with significant morbidity and mortality. ${ }^{1}$ Patients with congenital unilateral pulmonary vein atresia usually present in infancy or early childhood with recurrent pulmonary infections, dyspnea on exertion, or hemoptysis. ${ }^{2}$ Pulmonary vein atresia is associated with congenital heart defects in approximately $30 \%$ of the cases. ${ }^{3}$ Unilateral pulmonary vein atresia causes significant ventilation perfusion mismatch because hypoplasia of the pulmonary arteries on the affected side causes the development of systemic collaterals and subsequent left to right shunting. This patient's main complaint was progressive dyspnea on exertion and palpitations, but he denied other pulmonary complaints. Upon further questioning he had been told about an incidental finding on his chest $x$-ray as a teen; he had no evidence of congenital heart defects at that time.

Based on the current understanding of the mechanisms of atrial fibrillation, two major strategies can be considered for management: 1) catheter ablation of $\mathrm{AF}$ alone; or 2) a combination of anatomic ablation and electrogram-guided ablation. Patients undergoing AF ablation usually have cardiac imaging to evaluate pulmonary vein anatomy prior to the procedure. In our case, a preprocedural CT detected congenital absence of the left pulmonary veins. Due to the limited number of patients with this condition and limited information on catheter ablation in these cases, this procedure was deferred with a plan to assess pulmonary causes of the shortness of breath and dyspnea. This case demonstrates the clinical importance of preprocedural imaging prior to AF ablation.

Author Affiliation: Sharmila Sehli is a fellow in cardiac electrophysiology at The Warren Alpert Medical School of Brown University, Providence, Rhode Island. David M. Donaldson is a Clinical Assistant Professor of Medicine at The Warren Alpert Medical School of Brown University.

Received: $12 / 19 / 2014$

Accepted: $12 / 28 / 2014$

Reviewers: Scott Shurmur MD, Alejandro Perez-Verdia MD

Published electronically: 01/15/2015

Conflict of Interest Disclosures: none 


\section{REFERENCES}

1. Reller MD, McDonald RW, Gerlis LM, Thornerg KL. Cardiac embryology: basic review and clinical correlations. $J$ Am Soc Echocardiogr 1991; 4: 519-532.

2. Cullen S, Deasy PF, Tempany E, Duff DF. Isolated pulmonary vein atresia. Br Heart $J$ 1990; 63: 350-4.

3. Pourmoghadam KK, Moore JW, Khan M, Geary EM, Madan $\mathrm{N}$, Wolfson $\mathrm{BJ}$, et al. Congenital unilateral pulmonary venous atresia: definitive diagnosis and treatment. Pediatr Cardiol 2003; 24: 73-9. 